\title{
Challenges and Opportunities for Academic Journals to Serve the Older Population in Western Pacific Region
}

\author{
Hee-Won Jung ${ }^{1}$, Wee Shiong Lim $^{2}$, Matteo Cesari ${ }^{3}$, Tung Wai Auyeung ${ }^{4}$, Taro Kojima ${ }^{5}$, Hyuk Ga ${ }^{6}$, Ian D. Cameron ${ }^{7}$, \\ Jae-Young Lim ${ }^{8,9}$ \\ ${ }^{1}$ Division of Geriatrics, Department of Internal Medicine, Asan Medical Center, University of Ulsan College of Medicine, Seoul, Korea \\ ${ }^{2}$ Department of Geriatric Medicine, Institute of Geriatrics and Active Ageing, Tan Tock Seng Hospital, Singapore \\ ${ }^{3}$ Geriatric Unit, IRCCS Istituti Clinici Maugeri, University of Milan, Milan, Italy \\ ${ }^{4}$ Jockey Club Institute of Ageing, The Chinese University of Hong Kong, Hong Kong \\ ${ }_{5}^{5}$ Department of Geriatric Medicine, Graduate School of Medicine, The University of Tokyo, Tokyo, Japan \\ ${ }^{6}$ Institute of Geriatric Medicine, Incheon Eun-Hye Hospital, Incheon, Korea \\ ${ }^{7}$ John Walsh Centre for Rehabilitation Research, Northern Sydney Local Health District and University of Sydney, Kolling Institute, St Leonards, Australia \\ ${ }^{8}$ Department of Rehabilitation Medicine, Seoul National University Bundang Hospital, Seongnam, Seoul National University College of Medicine, Seongnam, \\ Korea \\ ${ }^{9}$ Institute on Aging, Seoul National University, Seoul, Korea
}

Corresponding Author:

Wee Shiong Lim, MBBS, MRCP, MHPE

Department of Geriatric Medicine,

Institute of Geriatrics and Active

Ageing, Tan Tock Seng Hospital, Annex

2, Level 3, 11 Jalan Tan Tock Seng,

Singapore 308433

E-mail: wee_shiong_lim@ttsh.com.sg

ORCID:

https://orcid.org/0000-0003-3975-7230

Received: November 15, 2021

Revised: November 19, 2021

Accepted: November 21, 2021
Annals of Geriatric Medicine and Research held its inaugural international editorial board virtual meeting on September 16, 2021, to brainstorm ideas for sustainable growth. This special article summarizes the key concepts obtained from the webinar proceedings, with further development of ideas from ensuing discussions occurring after the meeting. From the initial discussion points provided by eight editorial board members, including six presenters, email discussions further enriched these ideas to construct the current special article. The key points discussed were: impactful research and impact factors, international and Asian perspectives, and challenges to sustainable growth. The editors noted the existing gap between the impact factor and research impact as a challenge for the growth paths of regional journals. However, they agreed that persevering with impactful research would ultimately translate into parallel and gradual gains in impact, which is, therefore, consistent with the organic growth of the journal. Acknowledging challenges in navigating between unique Asian perspectives and international outlooks, the editors encouraged academic journals to serve as bridges linking international evidence with the richness of local perspectives. For sustainable growth, the editors suggested that journals may be forged into the academic ecosystems of the region, diversify value streams, and establish themselves as reputable brands in disciplines. By combining these discussions, we proposed the "IMPACT" strategy for journals on the growth path in the region, which stands for IMmersive user experience encompassing authors, reviewers, and readers; Pasteur's quadrant use-inspired research; Asia-Pacific context; Collaborative; and Translation to practice and policy.

Key Words: Publishing, Journals, Regional health planning, Aging, Cultural diversity

\section{INTRODUCTION}

As a journal focused on aging and age-related conditions, Annals of Geriatric Medicine and Research (AGMR) has aspired to address disparities by representing the rapidly growing older populations of Asian and emerging countries since its inception. ${ }^{1)}$ While the journal has experienced substantial growth in terms of volume and visibility, ${ }^{2)}$ challenges remain for AGMR in its quest to become a 
highly impactful arena for discussion. Therefore, to brainstorm ideas regarding design strategies for sustainable growth to adequately address the current challenges, AGMR held its inaugural international editorial board virtual meeting on September 16, 2021. The webinar included two lectures from the editors-in-chief of AGMR (JYL) and the Journal of Frailty and Aging (MC), four short talks from panelists, and fruitful discussion/brainstorming among editorial board members.

This special article summarizes the key concepts originating from the webinar proceedings, with further development of these ideas from ensuing discussions after the meeting. From the initial discussion points provided by eight editorial board members, including six presenters, email discussions further developed these ideas to construct the current special article. The key points discussed were: (1) impactful research and impact factors; (2) international and Asian perspectives; and (3) challenges to sustainable growth. Inputs from these eight participants were collected and drafted by HWJ and circulated for further enrichment. In particular, the authors aimed to encompass potential issues for academic journals on aging in general, not limiting the scope to AGMR itself. The participants felt that the accrued insights might be salient and potentially transferable to other journals in the Western Pacific region that may be experiencing similar challenges.

\section{CURRENT STATUS OF THE ANNALS OF GERIATRIC MEDICINE AND RESEARCH}

AGMR has undergone a significant transformation in recent years. Changing its language from Korean to English in 2016 heralded a significant shift in the focus of AGMR to grow from a national journal of geriatrics to a more international one, with a broader spectrum of topics spanning geriatrics and gerontology. In addition, noting the challenge of addressing the tremendous research gaps in Asian countries experiencing the rapid aging of their populations, AGMR sought to become a high-profile journal to first serve and support this region with evidence-based material. After being indexed in SCOPUS (2019) and PubMed Central (2020), submissions to the journal have doubled annually. The number of contributions from outside Korea has also skyrocketed. As AGMR remains a non-profit, open-access platform operating on funding support from the Korean Geriatrics Society, the editorial board has been overwhelmed by the growing volume of submissions and the need to maintain a reasonable turnaround time for each submitted manuscript. In this process, editorial board members face new potential conflicts and challenges in balancing the diverse goals for journal growth.

\section{IMPACTFUL RESEARCH AND IMPACT FACTOR}

Indeed, there has been an ongoing debate over whether the impact factor adequately represents the real impact of publications in journals. ${ }^{3)}$ The impact factor was initially established as a citation-based metric, a simple tool for ranking scientific journals from the standpoint of bibliometric influence." On the other hand, the Research Excellence Framework defined "impact" as "an effect on, change or benefit to the economy, society, culture, public policy or services, health, the environment or quality of life, beyond academia." ${ }^{, 5,6)}$ However, many countries and institutions have increasingly used the impact factor as the de facto metric in evaluating the track record of researchers. This environment creates pressure on researchers to study mature and crowded topics and is a disincentive to risky and potentially groundbreaking work requiring substantial lead time before obtaining citations. At the same time, the pressure to publish (for being cited) likely affects the general quality of the research output by prioritizing quantity. ${ }^{3)}$

This discrepancy between impact factor and research impact is particularly problematic in the Western Pacific region, where the fast pace of population aging underlines the pressing need for well-designed, impactful research that targets older persons. ${ }^{7}$ Unfortunately, the growth of academic journals (in addition to aging) in this region lags the pace of aging. There is a tendency for hysteresis-defined as a system with a rate-independent memory effect; ${ }^{8)}$ hence, the state of the system is dependent on its historyin the relationship between the visibility of academic journals (commonly represented by the impact factor) and the research impacts of their published papers. As researchers in many countries are rewarded by their quantifiable research output, impetus exists to submit better-quality papers to journals with higher impact factors.

Therefore, a chicken-and-egg cycle ensues for journals based in developing countries, with lower visibility (vis-à-vis the more prominent journals in aging), leading to fewer submissions.

Editors recognize and often have to grapple with the potential conflict between publishing potentially impactful research for the target population of the journal in the long run and soliciting manuscripts that are more helpful in attracting mid-term citations to increase the journal's impact factor. For example, although a smallscale hypothesis-generating study might have a substantial clinical impact on a specific population, the volume of researchers in the same milieu ${ }^{9)}$ that potentially cite the work may be less than the critical mass required to establish sufficient visibility for the study (especially in the context of the relentless flood of academic literature). Furthermore, the stringency of current clinical research standards in a publication that often preclude real-world studies, even 
in advanced societies, ${ }^{10)}$ may likewise prevent researchers in smaller organizations from establishing a viable research agenda based on clinical observations. In our experience, editors face a growing volume of manuscripts from developing countries that can be meaningful and novel when contextualized at the national level but may tend to be incomplete or marginal in terms of scientific rigor or generalizability (thus failing to find resonance in terms of perceived impact with high-quality bibliographic databases). Therefore, in the current stage of AGMR, the editors often find themselves choosing between Scylla and Charybdis in our roles as advocates of aging research in developing countries, fostering promising early-stage works (but with the tradeoff of less comprehensive scientific data) vis-à-vis more established but potentially less novel research.

Notwithstanding these challenges, there is agreement that, beyond its inherent significance, persevering with impactful research will ultimately translate into parallel and gradual gains in visibility, which is, therefore, consistent with the organic growth of the journal. Therefore, it is critical to resist the gentle nudge of hysteresis towards the false dichotomy of impactful research versus impact factor, especially when the journal's growth in visibility may lag behind the academic impact. Instead, we must steer the discourse towards an alignment between impactful research and impact factor. To this end, the editorial board comprising international scholars plays an important role in shaping research priorities, pushing the frontiers of knowledge, and cultivating impactful research that addresses specific gaps for populations around the region.

\section{INTERNATIONAL AND REGIONAL PERSPECTIVES}

Editors have also experienced a dilemma for a journal under development; that is, whether to steer the journal towards adopting a more international outlook or to retain its unique Asian perspectives. To be more global, journals aspire to reach a larger community of the target audience and play a more prominent role in guiding the direction of scientific research in general. ${ }^{9)}$ In contrast, this goal is less attainable for lower-profile regional journals. While more regional journals may attract more research specifically designed for a certain population, in some cases, they may, ironically, grapple with a lower volume of regional research submissions.

This issue is especially problematic for academic journals focused on the health and social issues of the older population. The feasibility and effectiveness of specific healthcare delivery models for the older population may be primarily affected by social structure, cultural perception, and economic resources, as previously noted in AGMR. ${ }^{1)}$ These factors might be less relevant for studying individual diseases with clearly defined biomedical definitions and therapeutics. For example, deprescribing medications in older adults with polypharmacy and frailty might be more critical in established medical systems with fee-for-service than in developing countries where seeking medical attention per se is barely accessible for most populations (and the number of excessive prescriptions is probably lower). "What matters" (i.e., one of the four cornerstones of the age-friendly health system framework ${ }^{11)}$ is also affected by the social characteristics of populations. While research diversity in aging is considered a pressing issue in the United States, ${ }^{12)}$ knowledge gaps among different societies have been less addressed globally.

The cultural characteristics of Asian countries have long been considered significant when dealing with the care issues of older persons. For instance, providing nutritional support through enteral access has been considered a default in many Asian societies, even in patients with advanced dementia, even though tube feeding in these patients is clinically controversial. ${ }^{12,13)}$ Similarly, a recent study suggested that socio-cultural factors pose barriers in initiating discussions about advance care planning in older persons with dementia. ${ }^{14)}$ Specifically, in Chinese culture, discussing matters related to death has been considered taboo. ${ }^{15)}$ In addition, it is not uncommon in Asian cultures for older people to leave end-oflife decisions to their family members. ${ }^{16)}$ Again, from the caregiving perspective, the traditional concept of filial piety has primarily served as a backbone for providing long-term caregiving for older adults with functional needs within the community. ${ }^{17)}$

However, the salience of filial piety and familial caregiving in many Asian societies is increasingly questioned as shrinking family size and urbanization make familial care of older adults less feasible and portend the growing unmet needs of social support for the older population. The evolving social structure and lifestyle of Asian countries might ultimately attenuate the importance of traditional cultural beliefs regarding the duty to care for older adults. Academic journals in this region may serve as bridges by linking international evidence with the richness of local perspectives, providing leadership to define research frontiers, and translating the findings of research studies to address the specific needs of target populations. ${ }^{18)}$ Altogether, journals would again serve to move beyond the limitations of a "false dichotomy" mindset by embracing a dualistic outlook of achieving both research impact and impact factor and harnessing the eclectic richness of international and regional perspectives.

\section{CHALLENGES TO SUSTAINABLE GROWTH}

Peer reviewers have long been considered the guardians of research quality. ${ }^{19)}$ Regional independent journals are usually operated by 
the voluntary support of editors and reviewers. Securing high-quality review standards for the growing volume of submitted manuscripts while simultaneously minimizing the turnaround time of peer review is particularly challenging in this context. Many regional scientific societies and organizations do not have sufficient resources to attract internationally renowned scholars. Even local scientists with competitive track records are less incentivized to support lower-profile journals. ${ }^{9)}$ As a significant proportion of manuscripts submitted to regional journals are of low quality (in terms of writing, editing, and scientific rigor), the increasing volume of submissions aggravates the editorial team's workload.

Moreover, the general volume of scientific literature has been growing, with many publishers choosing open access systems to maximize their volume, impact, and profit. Indeed, one of the most notable changes in the ecosystem of scientific publications over the last decade is the rise in open access publications. ${ }^{20)}$ Open access journals with extensive networks, standardized editing platforms, and existing brand premiums inherited from their flagship journals have enjoyed tremendous growth in terms of publication volume. However, unlike these journals, non-profit-independent journals backed by national scientific societies are experiencing ever-growing challenges in maintaining sustainable positive trajectories, both in quantity and quality.

Although attracting support from established scholars might be less feasible, regional journals can strategically leverage the uneven field of the research ecosystem. For example, while senior scholars have been primarily considered candidates for the role of peer reviewers, studies have shown that younger researchers tend to provide peer reviews of better quality. ${ }^{21,22)}$ Moreover, for junior researchers in their growing career path, regional journals with a strong relationship with local academic societies can serve as stepping stones for participation in core groups of researchers in the field and as editorial board members. In this ecosystem, regional journals can play a role as an arena for developing fresh ideas, innovative methodologies, and high-quality work by junior researchers and faculty members. To this extent, this ecosystem can be forged into a community of committed and motivated reviewers, regular contributors, and active readership. Thus, regional journals can serve as stepladders in research career development and as conduits for participation within the discipline. ${ }^{23)}$

Currently, most regional journals are financially supported by governments and national academic societies. Inherently, the limited amount of such funding restricts the expansion of publication volumes if these journals do not request article processing charges (APC). At the same time, imposing an APC is difficult for these journals, presumably experiencing a scarcity of high-quality manuscripts. Indeed, securing funding sources is a challenge for these journals, and a valley of death ensues before these journals get enough visibility to attract a sufficient number of authors to absorb the APC, similar to the experience of early-stage startup companies. ${ }^{24)}$ On the other hand, journals may expand their available resources by establishing sustainable business models that balance academic, professional, and financial considerations. These efforts can be facilitated by advances in technology, journal administration, and the agile activities of supporting staff. One example might be granting discount vouchers for reviewers that can be used as APCs for future submissions. This strategy incentivizes reviewers, simultaneously alleviating resistance to APC among authors. In addition, journals may hold educational conferences on research methodologies, writing, and other issues on academic publications to diversify sustainable value streams.

Another consideration for attaining a sustainable growth trajectory is the development of reputable journals in research fields. Both brand and impact factor matter in academic publications. Many high-quality manuscripts are submitted to recently started franchise journals without an impact factor by researchers anticipating that gaining visibility for the journal (and, thus, for their article) is just a matter of time. To build robust reputations for journals, editors and reviewers should provide consistent experiences for contributors. A good reputation is, ultimately, dependent on the time-honored values of trust, dependability, timeliness, and respect.

\section{CALL TO ACTION: THE "IMPACT" STRATEGY}

We discussed the challenges that regional journals on geriatrics and gerontology currently face in their growth trajectories in terms of research impact, regional characteristics, and sustainability. We agreed that dichotomies in these matters are not necessarily mutually exclusive and can be navigated through elaborations targeting the sweet spot of organic growth. We noted that these journals may serve as a critical platform for the academic ecosystem to provide immersive user experiences for members of academic disciplines in the region and attract meaningful and impactful research, thereby attaining visibility and completing a virtuous cycle of growth. Ironically, the disruption or cessation of research activities by the coronavirus disease 2019 (COVID-19) pandemic may represent a unique opportunity for regional journals to take advantage of well-conducted innovative research that will not reach the expected, high-level journals owing to incomplete data. ${ }^{25)} \mathrm{By}$ embracing a diverse array of possibilities, journals can steer towards pursuing research works that may translate into real-world practice in the region, from enriched perspectives driven by collaborations among professions and disciplines across regions. In navigating re- 


\begin{tabular}{|c|c|}
\hline IMmersive user experience & Forged into the research ecosystem of the discipline \\
\hline Pasteur's quadrant & Collaboration between profession and disciplines \\
\hline Collaborative & Apply knowledge from research to policy and practice \\
\hline Translational & Ascearch, pursuing both rigor and relevance \\
\hline
\end{tabular}

Fig. 1. The proposed "IMPACT" strategy, a sustainable growth path for Western-Pacific regional journals on aging and age-related conditions.

search and real-world applications, journals may adopt the concept of Pasteur's quadrant, pursuing both scientific excellence and usability in scientific research. ${ }^{26)}$ Putting these together, we call for an "IMPACT" strategy, an acronym for (1) IMmersive user experience encompassing authors, reviewers, and readers; (2) Pasteur's quadrant use-inspired research; (3) Asia-Pacific; (4) Collaborative; and (5) Translational (Fig. 1). We hope that by adopting this strategy, journals in the region may position themselves as the nexus of the value chain of research, from hypothesis-generating to policymaking and practice.

\section{ACKNOWLEDGMENTS}

\section{CONFLICT OF INTEREST}

The researchers claim no conflicts of interest.

\section{FUNDING}

None.

\section{AUTHOR CONTRIBUTIONS}

Conceptualization, HWJ, WSL; Project administration, HWJ, WSL, MC, TWA, TK, HG, IDC, JYL; Supervision, WSL; Writing-original draft, HWJ, WSL, MC; Writing-review and editing, HWJ, WSL, MC, TWA, IDC, JYL.

\section{REFERENCES}

1. Jung HW, Won CW, Lim JY. Annals of Geriatric Medicine and Research as a space of for developing research ideas into better clinical practices for older adults in emerging countries. Ann Geriatr Med Res 2019;23:157-9.

2. Lim JY, Jung HW, Ga H. Annals of Geriatric Medicine and Research indexed in PubMed Central: an important milestone toward the leading journal. Ann Geriatr Med Res 2020;24:155-6.

3. Alberts B. Impact factor distortions. Science 2013;340:787.

4. Simons K. The misused impact factor. Science 2008;322:165.
5. Research Excellence Framework. Decisions on assessing research impact. London, UK: Research Excellence Framework; 2011.

6. Penfield T, Baker MJ, Scoble R, Wykes MC. Assessment, evaluations, and definitions of research impact: a review. Res Eval 2014;23:21-32.

7. Kasai DT. Preparing for population ageing in the Western Pacific Region. Lancet Reg Health West Pac 2020;6:100069.

8. Visintin A. Differential models of hysteresis. Heidelberg, Germany: Springer; 1994.

9. Meneghini R. Emerging journals: the benefits of and challenges for publishing scientific journals in and by emerging countries. EMBO Rep 2012;13:106-8.

10. Lam HR, Chow S, Taylor K, Chow R, Lam H, Bonin K, et al. Challenges of conducting research in long-term care facilities: a systematic review. BMC Geriatr 2018;18:242.

11. Fulmer T, Mate KS, Berman A. The age-friendly health system imperative. J Am Geriatr Soc 2018;66:22-4.

12. Hattori Y, Kojima T, Komura H, Ura N, Akishita M. Percutaneous endoscopic gastrostomy tube removal for patients with advanced dementia: case series study. Ann Geriatr Med Res 2019; 23:27-30

13. Lee YF, Hsu TW, Liang CS, Yeh TC, Chen TY, Chen NC, et al. The efficacy and safety of tube feeding in advanced dementia patients: a systemic review and meta-analysis study. J Am Med Dir Assoc 2021;22:357-63.

14. Ali N, Anthony P, Lim WS, Chong MS, Poon EW, Drury V, et al. Exploring differential perceptions and barriers to advance care planning in dementia among Asian patient-caregiver dyads: a mixed-methods study. Int J Environ Res Public Health 2021;18: 7150 .

15. Hsu CY, O'Connor M, Lee S. Understandings of death and dying for people of Chinese origin. Death Stud 2009;33:153-74.

16. Lee MC, Hinderer KA, Kehl KA. A systematic review of advance directives and advance care planning in Chinese people from Eastern and Western cultures. J Hosp Palliat Nurs 2014;16:75- 
85.

17. Woo J. The myth of filial piety as a pillar for care of older adults among Chinese populations. Adv Geriatr Med Res 2020;2: e200012.

18. Lim WS, Wong CH, Ding YY, Rockwood K, Lien C. Translating the science of frailty in Singapore: results from the national frailty consensus discussion. Ann Acad Med Singap 2019;48:25-31.

19. Rennie D. Guarding the guardians: a conference on editorial peer review. JAMA 1986;256:2391-2.

20. Butler D. Investigating journals: the dark side of publishing. Nature 2013;495:433-5.

21. Evans AT, McNutt RA, Fletcher SW, Fletcher RH. The characteristics of peer reviewers who produce good-quality reviews. J Gen Intern Med 1993;8:422-8.

22. Stossel TP. Reviewer status and review quality: experience of the
Journal of Clinical Investigation. N Engl J Med 1985;312:658-9.

23. Bray R, Boon S. Towards a framework for research career development: an evaluation of the UK's Vitae Researcher Development Framework. Int J Res Dev 2011;2:99-116.

24. Al Natsheh A, Gbadegeshin SA, Ghafel K, Mohammed O, Koskela A, Rimpilainen A, et al. The causes of valley of death: a literature review. Proceedings of the 15th International Technology, Education and Development Conference (INTED); 2021 Mar 8-9; Virtual event.

25. Cesari M, Calvani R, Canevelli M, Aprahamian I, de Souto Barreto P, Azzolino D, et al. On Schrodinger's cat and evaluation of trials disrupted by the COVID19 pandemic: a critical appraisal.J Frailty Aging 2021;10:310-2.

26. Stokes DE. Pasteur's quadrant: basic science and technological innovation. Washington, DC: Brookings Institution Press; 2011. 